\title{
Kernicterus in an Adult Dog
}

\author{
Luciana Sonne', Djeison Lutier Raymundo', Bianca Santana de Cecco', Adriana da Silva Santos', \\ Caroline Argenta Pescador ${ }^{3}$, Eduardo Conceição de Oliveira' ${ }^{1}$ David Driemeier ${ }^{1}$
}

\begin{abstract}
Background: Kernicterus or bilirubin encephalopathy is a condition rarely observed in animal characterized by a yellowish discoloration of the central nervous system. It is a potentially fatal condition due to bilirubin neurotoxic effects caused by the increase of non-conjugated bilirubin pigment, which passes blood brain barrier and has been attributed to an imbalance between albumin and bilirubin levels. Intracellular bilirubin is toxic for cells and can cause decrease in protein synthesis, specially albumin, depression of cell respiration and cellular death. This paper describes kernicterus in a 2-year-old Great Dane female dog.

Case: Clinically, the animal showed apathy, lethargy, weight loss and jaundice, which progressed to vomiting and neurological signs characterized by loss of consciousness and eventually coma. Blood parameters were within normal range, except for high levels of alanine aminotransferase $(523 \mathrm{U} / \mathrm{L})$, suggesting a liver lesion. The animal was submitted to euthanasia due to the poor prognosis, and at post-mortem examination it showed dehydration and severe jaundice, especially oral, vaginal and ocular mucosas, subcutaneous tissue and blood vessels intima surface. The liver had an accentuated lobular pattern, and the stomach mucosa was reddened. Multiple petechiae were observed in the epicardium, as well as icterus in the blood vessels of the heart. Furthermore, the brain and cerebellum cortex, thalamic region and nuclei region of brainstem showed extensive icteric areas. Microscopically, the liver presented a mononuclear portal hepatitis, centrilobular necrosis and presence of yellowish pigments. The brain had neuronal necrosis, mild vacuolization of the white matter, perineuronal edema and Alzheimer type II astrocytes, while cerebellum showed Purkinje cells necrosis. Hepatic cooper measurement was within range values, and direct imunofluorescence for the detection of Leptospira sp. was negative.

Discussion: Kernicterus pathogenesis has been extensively studied, as the condition is commonly seen in neonatal humans. Diagnosis is based on gross and microscopic lesions in brain, which are consistent with bilirubin encephalopathy caused by the necrosis and degeneration of neurons. This condition is related to cases of intense hyperbilirubinemia, which exceeds the albumin binding capacity and, therefore, the excess of unconjugated bilirubin that can pass through the blood brain barrier. Liver disease causes deficient production of protein, especially albumin, decreasing the potential binding capacity to bilirubin, and consequently causing hyperbilirubinemia. In this case, the previously detected hepatic lesion suggested by liver enzymes increased, probably led to protein production dysfunction, causing hypoalbuminemia and hyperbilirubinemia. Unfortunately, albumin and bilirubin seric levels could not be measured. Decrease in albumin production along with the excess of unconjugated bilirubin caused the jaundice, and in cases like this one described, the blood brain barrier is compromised and the kernicterus occurs. Unconjugated bilirubin has negative effect in the glutamate uptake causing extracellular accumulation of it, which is consequently neurotoxic, causing necrosis and degeneration leading to a characteristic encephalopathy in animals with kernicterus. In this report, it was not possible to determine the primary hepatic disease, however this caused clinical neurotoxic disease, known as bilirubin encephalopathy.
\end{abstract}

Keywords: kernicterus, icterus, dog.

${ }^{1}$ Setor de Patologia Veterinária (SPV), Faculdade de Veterinária (FaVet), Universidade Federal do Rio Grande do Sul (UFRGS), Porto Alegre, RS, Brazil. ${ }^{2}$ Setor de Patologia Veterinária (SPV), Departamento de Medicina Veterinária (DMV) da Universidade Federal de Lavras (UFLA), Lavras, MG, Brazil. ${ }^{3}$ Departamento de Patologia Veterinária da Universidade Federal do Mato Grosso (UFMT), Cuiabá, MT, Brazil. CORRESPONDENCE: L. Sonne [lusonne@ yahoo.com.br - Tel.: +55 (51) 3308-6107]. Faculdade de Veterinária - UFRGS. Av. Bento Gonçalves n. 9090. Bairro Agronomia. CEP 91540-000 Porto Alegre, RS, Brazil. 


\section{INTRODUCTION}

Kernicterus, also known as nuclear jaundice or bilirubin encephalopathy, is a neurological condition characterized by bright yellow pigment deposition in the basal ganglia, hippocampus, subthalamic nucleus, cerebellum and spinal cord $[3,5,9]$. Kernicterus is caused by unconjugated bilirubin passage through the blood brain barrier [9]. Access of bilirubin to brain tissue has been attributed to imbalance between albumin and bilirubin in blood stream, resulting in high concentrations of unbound bilirubin [7]. Intracellular bilirubin is toxic for cells, and may cause a decrease in protein synthesis, respiration depression, glycolysis increase, changes in mitochondrial oxidative phosphorylation and cellular death $[2,8]$. It is a rare condition in animals, while in humans it has been reported in cases of erythroblastosis fetalis [6,7]. In children, bilirubin derived from erythrocyte destruction and from heme catabolism is not easily conjugated by immature liver, resulting in kernicterus $[1,8]$. This icterus can be caused by increase in production of bilirubin, deficiency in hepatic uptake, deficiency in bilirubin conjugation and increase in bilirubin enterohepatic circulation [2].

\section{CASE}

A 2-year-old Great Dane female dog was submitted for post-mortem examination, with clinical history informed by veterinarians in charge of apathy, jaundice and weight loss. Two weeks after the first examination, clinical condition worsened, progressing to vomiting and neurological signs. Hematological exams were within normal range; while biochemical exams revealed increase in alanine aminotransferase levels (ALT) $523 \mathrm{U} / \mathrm{L}$ (21-102 U/L), which decreased to $300 \mathrm{U} / \mathrm{L}$ after three days of treatment. Neurological signs progressed to seizures and coma, and, due to poor prognosis, euthanasia was elected. At necropsy, multiple organs were collected and fixed in $10 \%$ formalin. Subsequently, samples were processed routinely for histology, stained with hematoxylin-eosin (HE), and analyzed by optic microscopy. Frozen liver fragments were submitted to copper analysis. Kidney imprints were used for direct immunofluorescence test for Leptospira sp. This test was performed using a multivalent anti-Leptospira sp. antibody at 1:20 dilution, in humid chamber at $37^{\circ} \mathrm{C}$ for 30 minutes.

At necropsy, the ocular (Figure 1A), oral (Figure 1B), and vaginal mucosas, periosteum, blood vessels intima, and subcutaneous tissue were severely icteric. Additionally, liver had accentuated lobular pattern, as well as reddening of mucosa and serosa of urinary bladder. The heart showed multifocal hemorrhagic areas and icteric vessels. Renal cortex striations were evidentiated, and mucous membrane of the stomach was reddened. Brain was icteric on the cut surface in cerebral (Figure 1C) and cerebellar (Figure 1D) cortices, thalamus region and cerebellar and vestibular nuclei regions. Microscopically, liver had mononuclear portal hepatitis (Figure 2A), centrilobular hepatocytes necrosis with apoptotic bodies, and presence of yellow pigments. Kidney showed tubular degeneration, membranous glomerulonephritis and proteinaceous cylinders. The stomach mucosa had areas of mineralization in the mucosa. Urinary bladder showed mucosal was hemorrhagic. Cerebrum showed individual neuronal necrosis, mild white matter vacuolization, neuronal edema (Figure 2B) and Alzheimer type II cells, while cerebellum presented Purkinje cells necrosis. Direct immunofluorescence test for Leptospira sp. was negative and hepatic levels of copper were within normal range $(3.4 \mu \mathrm{g} / \mathrm{g})$.

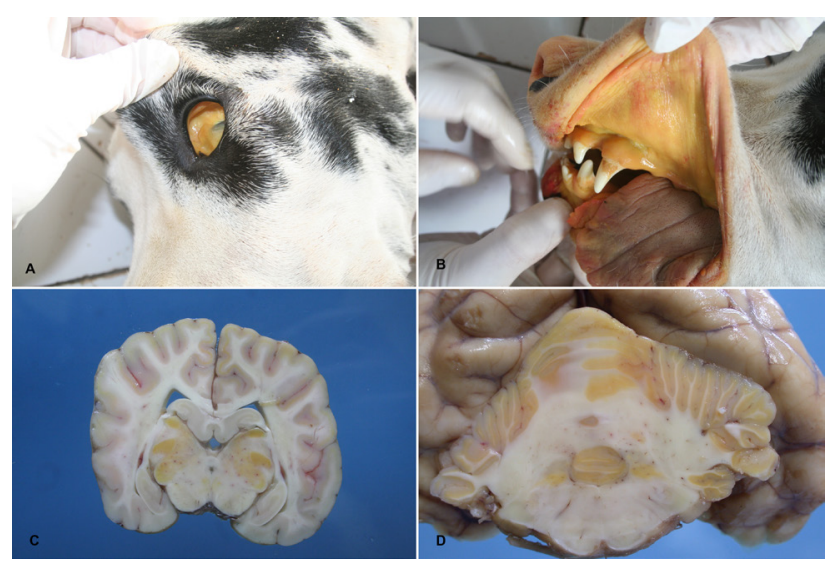

Figure 1. Kernicterus in a dog. A- Ocular mucosa severely icteric B- Oral mucosa severely icteric. C- Cerebral cut surface with severe jaundice. DCerebellar cut surface severely icteric.

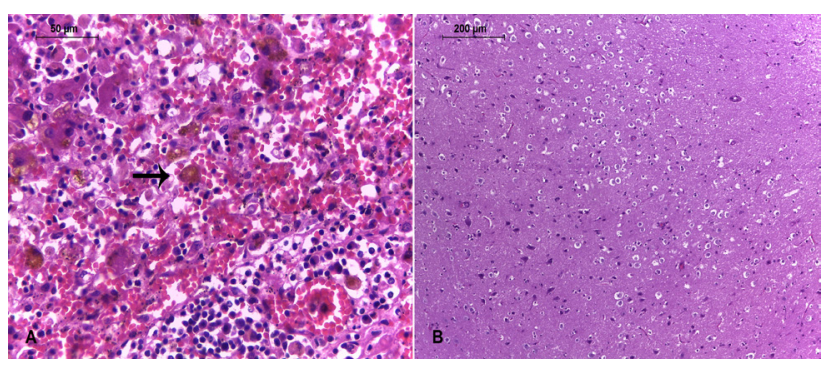

Figure 2. Kernicterus in a dog. A- Liver with mononuclear portal hepatitis, hepatocytes with apoptotic bodies and presence of yellow pigment (arrow) $\mathrm{HE}[\mathrm{Bar}=50 \mu \mathrm{m}]$. B- Brain with perineuronal edema $\mathrm{HE}[\mathrm{Bar}=50 \mu \mathrm{m}]$. 


\section{DISCUSSION}

Kernicterus diagnosis was based on gross and histological brain alterations. This condition is related to cases of intense hyperbilirubinemia, which exceeds the albumin binding capacity. Therefore, unconjugated bilirubin crosses blood brain barrier and causes kernicterus [9]. In this case, probably hepatic lesion was present for at least two weeks before death, and it caused bilirubin increase and albumin decrease. Hepatic lesions may cause reduced albumin production, leading to hypoalbuminemia, which increases unbound bilirubin concentration; however, unfortunately, levels of bilirubin and albumin were not analyzed in this case. Renal lesion may have been caused by likely increase in bilirubin, which in children has been reported to cause necrosis of renal tubules, intestinal mucosa and pancreatic cells due to its cellular toxicity [2,7]. Bilirubin is also a neurotoxic agent, causing negative effect in uptake of extracellular glutamate. It allows accumula- tion of glutamate and, consequently, an increased cell overexcitement, leading to apoptosis, necrosis and degeneration [4].

Kernicterus is a very rare condition in adult dogs, with a previously described case of kernicterus in an adult dog, which exhibited hepatic lesions of undetermined cause [9]. In humans, this condition may be observed in children suffering from erythroblastosis fetalis, however, it is rarely described in adults, with only two previous reports related to secondary hepatic disease [9]. Likewise, the cause of the hepatic lesion in this study could not be determined. However, the association of the clinical signs and the biochemistry analysis of liver enzymes it was possible to determinate a dysfunction of liver protein production and unconjugated bilirubin excess, which led to kernicterus.

Declaration of interest. The authors report no conflicts of interest. The authors alone are responsible for the content and writing of the paper.

\section{REFERENCES}

1 Ahlfors C. \& Herbsman O. 2003. Unbound bilirubin in a term newborn with kernicterus. Pediatrics. 111: 1110-1112.

2 Dennery P.A., Seidman D.S. \& Stevenson D.K. 2001. Neonatal hyperbilirubinemia. The New England Journal of Medicine. 344: 581-590.

3 Govaert P., Lequin M., Swarte R., Robben S., De Coo R., Weisglas-kuperus N., De Rijke Y., Sinaasappel M. \& Barkovich J. 2003. Change in globus pallidus with (pre) term kernicterus. Pediatrics. 112: 1256-1263.

4 Hanko E., Hansen T.W., ALmaas R, Lindstad J. \& Rootwelt T. 2005. Bilirubin induces apoptosis and necrosis in human NT2-N neurons. Pediatric Research. 57: 179-184.

5 Kumar V., Abbas A.K. \& Fausto N. 2005. Robbins and Cotran Pathologic Basis of Disease.7th edn. Philadelphia: Elsevier Saunders, 1525p.

6 Loynachan A.T., Williams N.M. \& Freestone J.F. 2007. Kernicterus in neonatal foal. Journal of Veterinary Diagnostic Investigation. 19: 209-212.

7 Ribeiro A.J.V., Batigália V.A., Kronka F.C. \& Rodriguez A.L.A.A.S. 2004. Kernicterus: relato de caso - breve revisão de literatura. Arquivos de Ciências da Saúde. 11: 55-58.

8 Rubin E. \& Killeen A.A. 2005. Doenças Genéticas e do Desenvolvimento. In: Rubin E., Gorstein F., Rubin R., Schwarting R. \& Strayer D. Rubin Patologia: Bases Clínicopatológicas da Medicina. 4.ed. Rio de Janeiro: Guanabara Koogan, pp.223-286.

9 Sangster C.R., Stevenson C.K., Kidney B.A., Montgomery D.L. \& Allen A.L. 2007. Kernicterus in an adult dog. Veterinary Pathology. 44: 383-385. 